\title{
Cyto-Histological Evaluation of Bronchial Brushing, Bronchial Washing and Bronchial Biopsy in Lung Tumours: A Prospective Study From Tertiary Care Centre in Southern Rajasthan.
}

\author{
Jigyasu Joshi* and Sunita Bhargava \\ Dept of Pathology,RNT Medical college,Udaipur.Rajasthan,India.
}

\begin{abstract}
Background: Lung cancer is the most frequently diagnosed major cancer in the world with a high mortality rate because most of the cases are diagnosed in advanced stage therefore early diagnosis of lung cancer is crucial in management. Examination of specimens obtained through flexible fiberoptic bronchoscope is an important and often the initial diagnostic technique performed in patients with suspected lung carcinoma. A study on cyto histological evaluation of bronchoscopic procedure samples was carried out to find out the efficacy of the procedures in lung tumours in southern Rajasthan.
\end{abstract}

Methods: Bronchoscopy was performed in total 100 patients with clinico- radiological suspicion of lung cancer. Bronchial brushing, washing and biopsy were taken wherever possible. Cytology and biopsy samples processed and slides prepared for examination.

Result: Overall sensitivity and specificity of bronchial brushing was $85.3 \%$ and $56.25 \%$ while for wash/BAL (bronchoalveolar lavage) the values were $78.34 \%$ and $59.26 \%$ respectively. Bronchial brushing had higher sensitivity for central as well as peripheral tumours while wash/BAL had higher sensitivity as well as specificity for peripheral tumours.

Conclusion: Bronchial brushing and wash/BAL has excellent sensitivity and good specificity and yields almost same information as biopsy. But regarding histological typing of lesions, biopsy is more effective than cytological study. Therefore, all these techniques may be used concurrently to diagnose the lung tumours.

Keywords: Bronchial Brushing, Bronchial Washing, Bronchial Biopsy, Fiberoptic Bronchoscopy, Lung Cancer.

\section{Introduction}

Lung cancer or bronchogenic carcinoma, is a malignant lung tumour characterized by uncontrolled cell proliferation in lung tissue. It is the most frequently diagnosed cancer currently and also the leading cause of the cancer related deaths across the world. ${ }^{[1,2]}$ Lung cancer was considered to be a rare entity in the beginning of 20th century but in past few years it has reached to almost epidemic proportions.

Cancer of the lung most often occurs between 40 to 70 years of age with only $2 \%$ cases occurring before 40 years of age. ${ }^{[3]}$ It is the commonest cause of death from cancer in males and the incidence is increasing dramatically in women also. The incidence of lung cancer is increasing globally and it has become the leading cause of death in most countries. The increasing incidence has been attributed to increase in smoking habit, changing life style, increased environmental pollution and availability of modern diagnostic modalities to detect lung cancer. ${ }^{[4,5]}$ In India, lung cancer constitutes 6.9 per cent of all new cancer cases and 9.3 percent of all cancer related deaths in both sexes. ${ }^{[6]}$

Lung cancer is a highly lethal disease because majority of the patients are diagnosed in advanced stage when it has become incurable. On the other hand, clinical outcome is significantly better in patients who are diagnosed at earlier stages when cancer is still confined within the lungs. Therefore, early diagnosis remains a key factor for favourable outcome. Traditionally diagnosis of lung cancer was based on findings of clinical examination and chest X-ray only. But over the years availability of bronchoscopy, newer radiological tools such as ultrasonography, CT scan and MRI and cytological and histological methods have revolutionized the diagnostic approach.

The introduction of flexible fiberoptic bronchofibroscope has significantly improved the diagnostic approach for lung cancer. The sampling techniques performed at flexible bronchoscopy for cytopathological examination of lung cancer include bronchial washing (BW), bronchoalveolar lavage (BAL) and bronchial brushing specimens while endobronchial forceps biopsy (EBB) and transbronchial forceps biopsy (TBB) can also be obtained for histopathological diagnosis. ${ }^{[7,8]}$ Bronchial biopsy along with immunohistochemistry form a gold standard for diagnosis of lung tumours but it is an invasive procedure and more expertise is required, while bronchial brushing $\&$ washing are comparatively simple and easy to perform. 
Therefore, cytological assessment of specimens of the respiratory tract using flexible FOB is the most commonly used technique for the diagnosis of lung cancer. Both bronchial washing and brushing used concurrently are effective in the diagnosis of neoplastic lesions of lung. These tend to preserve both the cells and their architectural arrangement. This technique can be used in conjunction with radiological and histological findings to give 100\% accuracy in the diagnosis of lung cancer.

\section{Materials And Methods}

This prospective analytic study was carried out in the department of Pathology, RNT medical college, Udaipur, Rajasthan on the Bronchoscopic procedures samples, received from clinico-radiologically highly suspicious patients of lung cancer attending the TB Hospital, Bari. The study was conducted after approval from Institutional Ethics Committee. Video bronchoscopy was performed in the department of Respiratory diseases and Tuberculosis. An informed consent was taken from the patients. Patient's demographic profile, smoking habits, clinical history, physical findings, chest $\mathrm{x}$-ray, CT thorax and bronchoscopic finding if done were recorded from bed headed tickets and personal interview. Patient's routine blood investigations were also recorded.

The samples were received in the department of pathology as already prepared air dried brushing smears; $20-30 \mathrm{ml}$ of bronchial washing fluid and bronchial biopsy in 10\% formalin. The washing fluid was centrifuged for five minutes at a rate of 1500 revolutions per minute and prepared into air-dried smears. The smears of brushing as well as washing were stained with May-Grunwald Giemsa. The biopsy tissues were processed as per standard procedure, $4-5 \mu \mathrm{m}$ thick sections were cut on microtome and stained by hematoxylin and eosin stain.

\section{Result}

Bronchoscopy and its allied procedures such as Bronchial brushing, Bronchial washing and Bronchial biopsy were carried out in 100 patients out of which 78 patients were diagnosed as having malignancy. Age of the patients ranged from 20-89 years with a mean of 59.4 years. Highest incidence of lung carcinoma was found between $61-70$ years comprising $36 \%$ (28) of total cases. Majority of the patients were male with a male female ratio of 12:1. 62 patients were smoker and 16 were non smoker giving a smoker: non smoker ratio of 3.88:1. Right lung was involved in $63 \%$ of cases while in 37\% cases Left lung was involved with middle lobe being most commonly involved. Squamous cell carcinoma was most common histological type (Figure 1) found in 50\% of cases followed by Adenocarcinoma (Figure 2) in $28 \%$ cases and Small cell carcinoma (Figure 3) in $19.4 \%$ cases (Table 1). Tumour was central in 56 cases (72\%) while in 22 cases $(28 \%)$ it was peripheral in location.

Morphological categorization of the cases by different techniques is shown in Table 2. All cases of lung carcinoma were categorized in to specific histological types by biopsy except 2 cases which showed normal histology on biopsy but diagnosed as squamous cell carcinoma by brushing and washing/BAL. Four cases labeled as non small cell carcinoma by brushing were found to be squamous cell carcinoma on biopsy. Out of 21 cases labeled as non small cell carcinoma on wash/BAL 14 cases were squamous cell carcinoma and 5 cases were adenocarcinoma on biopsy.

Overall sensitivity and specificity of bronchial brushing was $85.3 \%$ and $56.25 \%$ while for wash/BAL the values were $78.34 \%$ and $59.26 \%$ respectively (Table 3 ). Bronchial brushing had higher sensitivity for central as well as peripheral tumours while wash/BAL had higher sensitivity as well as specificity for peripheral tumours (Table 4).

Table 1: Distribution of lung carcinoma according to Histological types.

\begin{tabular}{|c|c|c|}
\hline Histological Type & No of cases & Percentage \\
\hline Squamous cell carcinoma & 39 & $28 \%$ \\
\hline Adenocarcinoma & 22 & $19.4 \%$ \\
\hline Small cell carcinoma & 15 & $1.3 \%$ \\
\hline Large cell carcinoma & 1 & $1.3 \%$ \\
\hline Sarcoma & 1 & \\
\hline Total & $\mathbf{7 8}$ & \\
\hline
\end{tabular}

Table 2: Categorization of all cases by different techniques.

\begin{tabular}{|c|c|c|c|}
\hline Types & Brushing & Washing/BAL & Biopsy \\
\hline Non small cell carcinoma & 4 & 21 & - \\
\hline Squamous cell carcinoma & 12 & 15 & 29 \\
\hline
\end{tabular}




\begin{tabular}{|c|c|c|c|}
\hline Types & Brushing & Washing/BAL & Biopsy \\
\hline Adenocarcinoma & 14 & 14 & - \\
\hline Large cell carcinoma & 1 & 1 & 13 \\
\hline Small cell carcinoma & 11 & - & 1 \\
\hline Sarcoma & - & 37 & 27 \\
\hline Non malignant & 19 & $\mathbf{1 0 0}$ & $\mathbf{8 7}$ \\
\hline Total & $\mathbf{6 1}$ & & \\
\hline
\end{tabular}

Table 3: Statistical evaluation of Brushing and Washing/BAL.

\begin{tabular}{|c|c|c|}
\hline Indices & Brushing & Washing/BAL \\
\hline True positive & 29 & 47 \\
\hline False positive & 7 & 11 \\
\hline True negative & 9 & 13 \\
\hline False negative & 5 & $78.34 \%$ \\
\hline Sensitivity & $85.3 \%$ & $59.26 \%$ \\
\hline Specificity & $56.25 \%$ & \\
\hline
\end{tabular}

Table 4: Comparison of indices for central and peripheral lung tumours

\begin{tabular}{|c|c|c|c|c|}
\hline \multirow{2}{*}{ Indices } & \multicolumn{2}{|c|}{ Central } & \multicolumn{2}{c|}{ Peripheral } \\
\cline { 2 - 5 } & Brushing & Washing/BAL & Brushing & Washing/BAL \\
\hline True positive & 21 & 35 & 5 & 4 \\
\hline False positive & 2 & 7 & 6 & 12 \\
\hline True negative & 3 & 6 & 1 & 2 \\
\hline False negative & 4 & $76.1 \%$ & $88.89 \%$ & $85.72 \%$ \\
\hline Sensitivity & $84 \%$ & $46.16 \%$ & $54.55 \%$ & $71.43 \%$ \\
\hline Specificity & $60 \%$ & & & 5 \\
\hline
\end{tabular}

\section{Discussion}

Lung cancer is currently the most frequently diagnosed major cancer in the world and the most common cause of cancer mortality worldwide. ${ }^{[9]}$ Previously bronchogenic carcinoma was considered to be infrequent in India, but in the recent past a trend of increase in its incidence has been noticed. ${ }^{[10]}$ Being a highly lethal disease lung cancer should be diagnosed at earliest possible stage before the lesion has reached the stage of a visible and palpable tumour. For early diagnosis different modalities are available but bronchoscopy is perhaps the most valuable tool for diagnosis of lung cancer. Cytological assessment of specimens of the respiratory tract is an important and often the initial diagnostic technique carried out in a patient with suspected malignant lung lesion. ${ }^{[1]}$ Among various bronchoscopic techniques, bronchial biopsy has the highest sensitivity for endobronchial malignant lesions. ${ }^{[12]}$ Bronchial biopsy has also been used as the gold standard diagnostic test to assess the efficacy of other cytologic techniques. ${ }^{[13]}$ Better diagnostic yield is often obtained when cytologic techniques are used together with bronchial biopsy. ${ }^{[14]}$
This study was carried out with the objective to assess the efficacy of bronchial brushing and washing in comparison with bronchial biopsy in central and peripheral lung tumours. Bronchoscopy was carried out on 100 patients out of which $78(78 \%)$ cases were confirmed as having lung cancer by bronchial biopsy. Squamous cell carcinoma was the most common type of lung cancer followed by adenocarcinoma and small cell carcinoma. These observations are in corroboration with findings of Navneet Singh et al [15] and Dhanalaxmi et al ${ }^{[16]}$ but differ from Bhat $\mathrm{N}$ et al ${ }^{[17]}$ and Ruchee Khandelwal et al ${ }^{[18]}$ who reported squamous cell carcinoma as the most common type followed by small cell carcinoma and adenocarcinoma. Over last few years there has been a shift of histological type of lung carcinoma towards adenocarcinoma worldwide. ${ }^{[19]}$ Our study is at variance to western literature where incidence of adenocarcinoma has surpassed squamous cell carcinoma ${ }^{[20]}$ and its incidence is increasing rapidly all over the world. Studies in India by Prabhat Singh et al ${ }^{[21]}$ and Raj SM et al ${ }^{[22]}$ also reported adenocarcinoma as the most common histological type. 
There is wide variability in results of different studies regarding efficacy of different techniques. Some authors reported higher sensitivity and specificity of bronchial brushing while others have observed higher values for bronchial wash/BAL. Results are affected by several factors such as technique and expertise of the pulmonologist doing bronchoscopy, type of instruments being used, technique for retrieval and processing of cytological specimen and experience of reporting cytopathologist. In present study we observed sensitivity of bronchial brushing and washing/BAL $85.3 \%$ and $78.34 \%$ with specificity $56.25 \%$ and $59.26 \%$ respectively which is similar to the results by Shagufta TM et al ${ }^{[23]}$ who reported sensitivity of $82.1 \%$ and $74.5 \%$ with higher specificity of $72.7 \%$ and $62.5 \%$ respectively. Studies by M Ahmad et al ${ }^{[24]}$ and D Raiza et al ${ }^{[25]}$ show sensitivity of $80.5 \%$ and $80.5 \%$ for brushing and wash/BAL combined, with higher specificity of $96.6 \%$ and $92.85 \%$ while Dhanalaxmi et al ${ }^{[16]}$ observed higher sensitivity of $90.9 \%$ and specificity of $68.97 \%$ which is close to the current study. For centrally located tumours we found sensitivity and specificity for bronchial brushing of $84 \%$ and $60 \%$ while for wash/BAL $76.1 \%$ and $46.16 \%$ respectively while in peripheral tumours the recorded values for brushing were $88.89 \%$ and $54.55 \%$ while for wash/BAL the values were $85.72 \%$ and $71.43 \%$ respectively. Sensitivity and specificity are affected by the number of false positive and false negative results in any study. False positive results can be due to misinterpretation of the smears by the cytologist due to cellular changes in chronic inflammatory disorders such as chronic pneumonia (atypical histiocytes), tuberculosis (epitheloid cells), pneumonitis (misinterpretation of cuboidal alveolar cells as small cell carcinoma), squamous metaplasia and alveolar cell polymorphism in lung fibrosis. On the other hand, false negative results can be due to superadded inflammation and non representative material or hypocellular aspirates in some cases.

\section{Conclusion}

Thus after analyzing the results of the present study it was concluded that bronchial brushing and wash/BAL has excellent sensitivity and fairly good specificity. It yields almost same information as biopsy and is particularly useful in patients with evidence of obstruction or risk of haemorrhage. It is quite safe and economical. But regarding histological typing of lesions, biopsy is more effective than cytological study. Therefore, all these techniques may be used concurrently to diagnose the lung tumours.

\section{Acknowledgements}

We acknowledge the immense help received from the scholars whose articles are cited and included in references of this manuscript.

\section{Reference}

1. Brambilla E, Travis WD. Lung cancer. In: Stewart BW, Wild CP (eds) World Cancer Report. World Health Organization, Lyon, 2014; 489-508.

2. Siegel R, Ma J, Zou Z, Jemal A. Cancer statistics, 2014. CA. Cancer J. Clin 2014; 64 (1): 9-29.

3. Jemal A, Bray F, Center MM, Ferlay J, Ward E, Forman D. Global cancer statistics. CA Cancer J Clin. 2011;61(2):69-90.

4. The Health Consequences of Smoking: A Report of the Surgeon General. U.S. Department of Health and Human Services, Public Health Service, Centers for Disease Control and Prevention, Washington, DC 2004. CDC Publication No. 7829.

5. Magrath I, Litvak J. Cancer in developing countries opportunity and challenge. J. Nat. Cancer, Itrt. 1993;85(11):862-74.

6. Prabhat Singh Malik \& Vinod Raina. Editorial: Lung cancer: Prevalent trends \& emerging concepts. Indian J Med Res 141, January 2015,pp 5-7.

7. Bodh A, Kaushal V, Kashyap S, Gulati A. Cytohistological correlation in diagnosis of lung tumors by using fiberoptic bronchoscopy: Study of 200 cases. Indian J Pathol Microbiol. 2013;56:84-8.

8. Kotadia TP, Jasani JH, Vekaria PN. Comparison of bronchial biopsy, broncho alveolar lavage (BAL), brush cytology and imprint cytology in suspected cases of lung cancer International Journal of Biomedical and Advance Research. 2013:04(09):580-4.

9. Brambilla E, Travis WD, Colby TV, Corrin B, Shimosato Y. The new world health organization classification of lung tumours. Eur Respir J. 2001;18:1059-68.

10. Behera D, Balamugesh T. Lung cancer in India. Indian $J$ Chest Dis Allied Sci 2004;46:269-81.

11. Guidelines of the Papanicolaou Society of Cytopathology for the examination of cytologic specimens obtained from the respiratory tract. Papanicolaou Society of Cytopathology Task Force on Standards of Practice. Diagn Cytopathol 1999;21:61-9.

12. Schreiber G, McCrory DC. Performance characteristics of different modalities for diagnosis of suspected lung cancer: Summary of published evidence. Chest 2003;123:115S-28.

13. Gaur DS, Thapliyal NC, Kishore S, Pathak VP; Efficacy of broncho-alveolar lavage and bronchial brush cytology in diagnosing lung cancers; Journal of Cytology 2007; 24 (2) : 73-77.

14. Rivera MP, Detterbeck F, Mehta AC, American College of Chest Physicians. Diagnosis of lung cancer: The guidelines. Chest 2003; 123:129S-36.

15. Navneet S, Ashutosh NA, Dheeraj G, Digambar B, Surinder KJ. Quantified smoking status and non-small cell lung cancer stage at presentation: analysis of a North Indian cohort and a systematic review of literature. J Thorac Dis 2012;4(5):474-484. 
16. Dhanalakshmi S, Priyadharshini M, Narmadha, Manibharathi R. Cytohistological Correlation of Fibreoptic Bronchoscopy Specimens with Added Value of Immunohistochemistry in Diagnosis of Lung Tumors. IOSR Journal of Dental and Medical Sciences. Volume 16, Issue 8 Ver. I (Aug. 2017), PP 18-21

17. Nazia B, Mir JN, Humaira B, Nusrat B, Summiya F, Kaneez F, Khalil MB. Correlation of bronchial biopsy with bronchoalveolar lavage in lung malignancies. Int J Res Med Sci. 2016 Feb;4(2):428-435.

18. Ruchee K, Deepti A, Akriti A and Kriti A. Usefulness of Bronchial washings in diagnosing lung malignancy. International Journal of Biomedical and Advance Research 2018; 9(3): 80-84.

19. Valaitis J, Warren S, Gamble D (1981). Increasing incidence of adenocarcinoma of the lung. Cancer,47,1042-6.

20. Wahbah M, Boroumand N, Castro C, El-Zeky F, Eltorky M. Changing trends in the distribution of the histologic types of lung cancer. A review of 4, 439 cases. Ann Diagn Pathol. 2007;11:89.
21. Prabhat SM, Mehar CS, Bidhu KM, N K Shukla, SVS Deo, Anant M, Guresh K, Vinod R. Clinico-pathological profile of lung cancer at AIIMS: A changing paradigm in India. Asian pacific journal of cancer prevention, Vol 14,2013.

22. Mathan RS., Sowmiya M. Does a routine post brush bronchial wash increase the yield in diagnosis of lung cancer? Int J Res Med Sci. 2017 Jul;5(7):2878-2882.

23. Shagufta TM, Ghadeer AM. Diagnostic value of bronchial wash, bronchial brushing, fine needle aspiration cytology versus combined bronchial wash and bronchial brushing in the diagnosis of primary lung carcinomas at a tertiary care hospital. Biomedical Research (2015) Volume 26,Issue 4.

24. M. Ahmad, S. Afzal, W. Saeed, A. Mubarik, N. Saleem, S. A. Khan, S. Rafi. Efficacy of Bronchial Wash Cytology and its correlation with Biopsy in Lung Tumours. JPMA $54: 13 ; 2004$.

25. Raiza D, Rout S, Reddy KP, Ramalaxmi P, Prithvi BK, Harikishan KS. Efficacy of bronchial wash and brush cytology and its correlation with biopsy in lung lesions. IJHRMIMS. 2014 Oct;1(1): 21-24.

*Corresponding author:

Dr. Jigyasu Joshi, 107 A Shubh Labh Appartment, New Keshav Nagar, Udaipur, Rajasthan.

Phone: +91 9461385242

Email: jigyasudoc@gmail.com

Financial or other Competing Interests: None. 